\title{
Insolubility Theorems and EPR Argument
}

\author{
Guido Bacciagaluppi*
}

19 May 2012

\begin{abstract}
I present a very general and simple argument — based on the no-signalling theorem - showing that within the framework of the unitary Schrödinger equation it is impossible to reproduce the phenomenological description of quantum mechanical measurements (in particular the collapse of the state of the measured system) by assuming a suitable mixed initial state of the apparatus. The thrust of the argument is thus similar to that of the 'insolubility theorems' for the measurement problem of quantum mechanics (which, however, focus on the impossibility of reproducing the macroscopic measurement results). Although I believe this form of the argument is new, I argue it is essentially a variant of Einstein's reasoning in the context of the EPR paradox — which is thereby illuminated from a new angle.
\end{abstract}

\section{Von Neumann's insolubility argument}

In 1932, J. von Neumann provided one of the earliest and most famous discussions of the measurement process, in chapter VI of his Mathemati-

*Department of Philosophy, University of Aberdeen, and Institut d'Histoire et de Philosophie des Sciences et des Techniques (CNRS, Paris 1, ENS). Address for correspondence: Department of Philosophy, University of Aberdeen, Old Brewery, High Street, Aberdeen AB24 3UB, Scotland, U.K. (e-mail: g.bacciagaluppi@abdn.ac.uk). 
cal Foundations of Quantum Mechanics (von Neumann 1932 ${ }^{1}$ ). After setting up the problem and discussing composite systems in the first two sections, von Neumann begins his crucial section 3 with an argument 'to exclude an often proposed possibility for explaining the statistical character of process 1' (p. 437), where von Neumann's 'process 1' (which he defines on p. 351) is the collapse upon measurement of the quantum state of a system.

The kind of putative explanation von Neumann has in mind appears to be the following. ${ }^{2}$ Assume that the measuring apparatus is initially in some appropriate mixed state $\rho$. For any initial (pure) state $|\psi\rangle\langle\psi|$ of the system, the final state evolving unitarily from the initial $|\psi\rangle\langle\psi| \otimes \rho$ will also be mixed:

$$
U(|\psi\rangle\langle\psi| \otimes \rho) U^{-1}
$$

Could our initial ignorance of the apparatus state explain the final mixture of apparently collapsed states? This idea (before he proceeds to refute it) is formulated by von Neumann as follows (pp. 437-438):

Let $I$ be the observed system, $I I$ the observer. [...] The statistical character comes from the fact that while indeed $I$ was in a homogeneous state ${ }^{3}$ before the measurement, $I I$ instead was a mixture - and the character of mixture of $I I$ has 'infected' $I+I I$ during the course of the interaction, in particular has made a mixture of the projection in $I$. That is: the result of the measurement is indeterminate because the state of the observer before the measurement is not precisely known. It might be conceivable that such a mechanism works $[\ldots]$

Or is it? To fix ideas, take the case of an ideal measurement of electron spin in some given direction. We are asking whether we can construct a

\footnotetext{
${ }^{1}$ Page references are to the 1955 translation (which I have mostly tacitly amended).

${ }^{2}$ It is less apparent to whom von Neumann attributes such an explanation. Who are the ones who 'often proposed' it? (See also the discussion in section 4.)

${ }^{3}$ The 1955 translation has ' $I$ was in a (unique) state', but the German 'einheitlich' is used by von Neumann explicitly as a synonym for 'rein', i.e. 'pure', in which case it is generally translated as 'homogeneous' (see e.g. the definition of a 'homogeneous or pure' expectation functional on p. 307). Clearly, von Neumann here means a pure state, represented by a projection $|\psi\rangle\langle\psi|$.
} 
Hilbert space $\mathcal{H}$ describing an apparatus, ${ }^{4}$ together with a unitary evolution $U$ for the total system of electron and apparatus, an initial apparatus state $\rho$, and a finest ignorance-interpretable decomposition

$$
\rho=\int_{\Lambda} w_{\lambda} \rho_{\lambda} d \lambda
$$

of $\rho$, such that for any initial state

$$
|\psi\rangle=\alpha|+\rangle+\beta|-\rangle
$$

of the system, there is a partition $\Lambda=\Lambda_{+} \cup \Lambda_{-}$, generally dependent on $|\psi\rangle$, such that

$$
\int_{\Lambda_{+}} w_{\lambda} d \lambda=|\alpha|^{2}, \quad \int_{\Lambda_{-}} w_{\lambda} d \lambda=|\beta|^{2},
$$

and for all $\lambda \in \Lambda_{ \pm}$,

$$
U\left(|\psi\rangle\langle\psi| \otimes \rho_{\lambda}\right) U^{-1}=| \pm\rangle\langle \pm| \otimes \sigma_{ \pm}
$$

where the $\sigma_{ \pm}$represent distinct readings of the apparatus.

Von Neumann's own argument to the contrary is that, assuming the relevant decomposition of the final state (1) to be of the form

$$
\sum w_{n}\left|\psi_{n}\right\rangle\left\langle\psi_{n}|\otimes| \varphi_{n}\right\rangle\left\langle\varphi_{n}\right|
$$

(where the $\left|\psi_{n}\right\rangle$ denote the eigenstates of the measured observable, and the $\left|\varphi_{n}\right\rangle$ are some orthonormal set of apparatus states ${ }^{5}$ ), the weights in this decomposition are uniquely determined by the weights in the orthogonal decomposition of the initial apparatus state $\rho$, and are thus independent of the state $|\psi\rangle$ (in our example, this is inconsistent with (4)). This is true of any decomposition of this form, since the weights are unique up to ordering (pp. 438-439).

\footnotetext{
${ }^{4}$ Since the evolution of the total system is unitary, the total system needs to be closed, and we need to include in the apparatus anything with which the system will interact directly or indirectly. That is, we need to include in the apparatus also all the relevant parts of the environment. For the sake of brevity, however, we shall usually just talk about the 'apparatus'.

${ }^{5}$ Presumably, the microscopic states of the apparatus corresponding to different readings need to be orthogonal, but will not form a basis of the (very high-dimensional) apparatus Hilbert space.
} 
To avoid confusion and for further reference (in particular when we discuss Brown (1986) in the next section), it will be useful to distinguish between three different claims, only one of which is von Neumann's target. Let us call the triple $(\mathcal{H}, U, \rho)$ a measurement scheme, for brevity. Then the three claims are:

(i) For any $|\psi\rangle$ there exist a measurement scheme $(\mathcal{H}, U, \rho)$ and a finest ignorance-interpretable decomposition of $\rho$ that explain the phenomenological collapse, in the sense that there is a partition of the elements of the decomposition leading with the probabilities (4) to the collapsed states (5).

(ii) There exists a measurement scheme $(\mathcal{H}, U, \rho)$ such that for any $|\psi\rangle$ there exists a finest ignorance-interpretable decomposition of $\rho$ that explains the phenomenological collapse in the above sense.

(iii) There exist a measurement scheme $(\mathcal{H}, U, \rho)$ and a finest ignoranceinterpretable decomposition of $\rho$ that explain the phenomenological collapse for any $|\psi\rangle$.

Claim (i) is clearly not von Neumann's target, because it is trivially true (and thus impossible to rule out). Furthermore, it would be 'conspiratorial', in the sense that the measurement scheme is allowed to depend on the initial state of the system to be measured, but one's intuition is that the apparatus cannot know in advance what state of the system to expect. ${ }^{6}$

Claim (ii) is a stronger claim (thus one can conceive of trying to rule it out), but it does not seem to correspond to von Neumann's verbal statement of the problem. And indeed, since the finest ignorance-interpretable decomposition of $\rho$ fixes the set of possible initial apparatus states that are invoked to explain the different measurement results, letting this decomposition depend on the state $|\psi\rangle$ of the system to be measured means that in general the actual initial state of the apparatus will depend on $|\psi\rangle$. But that would be equally conspiratorial.

\footnotetext{
${ }^{6}$ It is interesting to consider whether such apparently conspiratorial dependencies might be explainable in retrocausal terms (see e. g. Price 1996), but this is clearly not a strategy von Neumann was considering.
} 
Thus, von Neumann's target must, indeed, be claim (iii), as sketched above. It is the strongest claim, and the easiest to rule out. It should be noted, however, that von Neumann's assumption that the ignoranceinterpretable decomposition of the final state is of the form (6) essentially constrains this decomposition uniquely, so that allowing the decomposition to depend on $|\psi\rangle$ does not make the claim more general. That is, in the restricted context of von Neumann's own proof, the distinction between claims (ii) and (iii) is not significant. It only becomes so in the context of later generalisations of von Neumann's result, and it is to these we now turn.

\section{Insolubility theorems}

Over the years, several physicists and philosophers have proved various generalisations of von Neumann's result, which are collectively known in the foundations literature as 'insolubility theorems' for the measurement problem of quantum mechanics. ${ }^{7}$ There are some differences that are worth spelling out, however, between von Neumann's original argument and most of the later ones.

First of all, recall that the phenomenological description of (ideal) measurements in quantum mechanics includes two aspects: (1) measurement results are definite, and distributed statistically according to the Born rule; and (2) the system is collapsed into a corresponding eigenstate of the measured observable. Different insolubility theorems focus on different aspects of this phenomenology. All more recent theorems focus on the impossibility of reproducing (the statistics of) definite pointer readings. Instead, von Neumann's formulation of the theorem is in terms of reproducing (the statistics of) the collapse of the state of the system. The focus on collapse may be

\footnotetext{
${ }^{7}$ A probably not quite exhaustive list includes Wigner (1963), d'Espagnat (1966), Earman and Shimony (1968), Fine (1970), d'Espagnat (1971, Sections 14-1, 14-2, 15-3 and 15-5), Fehrs and Shimony (1974), Shimony (1974), Brown (1986), Busch and Shimony (1996), Stein (1997), and Bassi and Ghirardi (2000). Most of these theorems concern measurements of discrete projection-valued (PV) observables (possibly including errors and disturbance). The only exceptions are the two theorems in Busch and Shimony (1996), which establish insolubility also for measurements of continuous PV observables and for measurements of arbitrary positive-operator-valued (POV) observables, respectively. (For a general reference to POV observables, see Busch, Grabowski and Lahti (1995).)
} 
inessential in von Neumann's proof, ${ }^{8}$ but we shall be making essential use of it for our own proof in the next section.

Another difference between von Neumann's theorem (or at least his verbal statement of the problem) and most of the later theorems was pointed out by Brown (1986). Brown noted that (with one exception) all authors writing on insolubility after von Neumann and up to 1986 had focused on showing the impossibility of the following type of claim:

(ii') There exists a measurement scheme $(\mathcal{H}, U, \rho)$ such that for all $|\psi\rangle$ there is a decomposition of the final state of system and apparatus in which the apparatus has a macroscopically well-defined reading. ${ }^{9}$

Ruling out (ii') would seem a straightforward way of ruling out that the phenomenology of measurements can be recovered in a model consisting of a unitarily interacting system and apparatus (and indeed it does). However, it is important to keep in mind that such a model would provide a solution to the measurement problem if and only if the relevant decomposition of the final state is ignorance-interpretable. The conceptual clarity of von Neumann's approach lies precisely in his asking whether we can trace the origin of any ignorance in the final state of the total system to ignorance in the initial state of the apparatus. If we do so in the case of (ii') (at least if we assume that the initial state of the system is pure ${ }^{10}$ ), we see that (ii') is equivalent to the claim we labelled as (ii) in the last section. That is, we are allowing the

\footnotetext{
${ }^{8}$ Von Neumann assumes the orthogonality of the states $\left|\psi_{n}\right\rangle \otimes\left|\varphi_{n}\right\rangle$ in the relevant decomposition of the final state. Thus, he could have easily phrased his result in terms of the orthogonal pointer states $\left|\varphi_{n}\right\rangle$, rather than the collapsed states $\left|\psi_{n}\right\rangle$ of the system. Indeed, if one does so, von Neumann's proof generalises to the case of disturbing measurements (i. e. where the system is collapsed into some disturbed states $\left|\tilde{\psi}_{n}\right\rangle$ ). As a matter of fact, von Neumann's proof generalises even to the case of approximate measurements (where the final distribution is only approximately given by the squared moduli of the coefficients in the initial state $|\psi\rangle)$ — since even in an approximate measurement one requires some dependence of the final distribution on the initial state of the system.

${ }^{9}$ Depending on the theorem in question, perhaps the triple $(\mathcal{H}, U, \rho)$ satisfies some further minimal constraint to justify labelling it indeed a 'measurement scheme'; perhaps the result holds only for almost all $|\psi\rangle$; perhaps the apparatus has a definite reading only with probability close to 1 ; etc.

${ }^{10}$ If the initial state of the system is not pure, then in general we can no longer trace an ignorance in the final state to ignorance of the initial apparatus state alone.
} 
relevant decomposition of the state of the apparatus (describing the possible states the apparatus might be in) to depend on the state of the system to be measured, which, as we said, is a conspiratorial scenario.

Thus, while ruling out (ii') rules out also our claim (iii), and thus proves the insolubility of the measurement problem also in von Neumann's sense, Brown wonders whether the focus on claims of type (ii') might not be conceptually misleading, ${ }^{11}$ and in any case focusing on (iii) simplifies the proofs. Brown singles out Fine (1970) as (implicitly) proving the weaker and conceptually neater result, and presents his own proof as a more explicit version of the one by Fine.

The more recent proof by Bassi and Ghirardi (2000) also falls into this category, and is of disarming simplicity and generality. The standard formulations of the measurement problem point out that for an initial superposition of eigenstates of the measured observable (e.g. electron spin, as in (3)), the final state of system and apparatus will be a superposition of states in which the apparatus has different readings. Bassi and Ghirardi reason in close analogy to this standard argument. They assume that the relevant decomposition of the initial mixed state of the apparatus (and environment) is such that, for the vast majority of components (a fraction $1-\varepsilon$ ), a measurement performed on the system in an eigenstate of spin will yield a final state of system and apparatus (and environment) that counts as including a macroscopic reading of the corresponding result. They also assume that any two distinct macroscopic states of system and apparatus (and environment) are at least approximately orthogonal. Given these assumptions, Bassi and Ghirardi point out that for a generic initial superposition (3), the vast majority of components of the initial state of the apparatus (a fraction $1-2 \varepsilon$ ) lead to a final state of system and apparatus (and environment) that fails to be approximately orthogonal to any of the states that include a macroscopic reading of spin up or down, and that therefore fails to be macroscopically well-defined.

\footnotetext{
${ }^{11}$ One could be charitable of course, and point out that it might be of independent interest to rule out also putative models with such 'conspiratorial' dependencies on $|\psi\rangle$ of the relevant decomposition of $\rho$.
} 


\section{$3 \quad$ Insolubility from no-signalling}

The purpose of this paper is to present an equally simple and general proof of insolubility, but one that focuses specifically on the impossibility of reproducing the collapse of the state of the system, rather than the definiteness of measurement results. I shall show that, even for a single state $|\psi\rangle$ of the system, if this state is an entangled state of two spatially separated subsystems (say, the singlet state of an EPR pair), it is impossible to reproduce the collapse of both particles by unitarily interacting locally with only one particle.

This may sound obvious, and I hope it will be obvious on reflection. One way to see this is by considering the Bell inequalities. The microscopic state in the relevant decomposition of $\rho$ plays the role of an apparatus hidden variable, and the unitary evolution is deterministic. From Bell's original analysis (Bell 1964), we know that any deterministic hidden variables theory that reproduces the violation of the Bell inequalities would allow for instantaneous signalling if one could in principle control the hidden variables. But in the putative models under consideration the hidden variables are just quantum states that are elements of the relevant decomposition of $\rho$, and the dynamics is just unitary evolution. And there is no way of signalling instantaneously by implementing on one side of an EPR setup local unitary interactions, even with suitably controlled quantum states. Thus the models cannot reproduce violation of the Bell inequalities, and a fortiori they must be unable to reproduce the collapse and Born rule in general.

We have, however, no need of a detour via the Bell inequalities. The same conclusion can be established by direct means, and could have been derived in essence already in the $1930 \mathrm{~s}^{12}$ The details are as follows.

Suppose we prepare a pair of electrons in an entangled state $|\psi\rangle$, say, the singlet state, and we perform a measurement of spin in a given direction on one of the electrons (say, Alice's). We now apply (2)-(5) to this case. We assume there is a decomposition of $\rho$,

$$
\rho=\int_{\Lambda} w_{\lambda} \rho_{\lambda} d \lambda
$$

\footnotetext{
${ }^{12}$ See also footnote 18 below.
} 
and a partition

$$
\Lambda=\Lambda_{+} \cup \Lambda_{-}
$$

with

$$
\int_{\Lambda_{+}} w_{\lambda} d \lambda \approx \int_{\Lambda_{-}} w_{\lambda} d \lambda \approx \frac{1}{2}
$$

(the statistics of results will actually not enter our argument, so that these values are immaterial for our purposes), and such that for all $\lambda \in \Lambda_{ \pm}$,

$$
U\left(|\psi\rangle\langle\psi| \otimes \rho_{\lambda}\right) U^{-1}=|\mp\rangle\langle\mp|\otimes| \pm\rangle\langle \pm| \otimes \sigma_{ \pm}
$$

the $\sigma_{ \pm}$representing distinct readings of Alice's apparatus.

More generally, we shall assume that the total system evolves to states that are indistinguishable from the states on the right-hand side of (10), even though they might contain some classical or quantum correlations. These states need not be the same on each run of the experiment, as long as they are recognisable as indicating the given result. We can also allow for slight variation (even co-variation) from one run of the experiment to another both in the initial states $|\psi\rangle$ and in the measurement scheme $(\mathcal{H}, U, \rho)$, as long as for the correct proportion of runs a condition of the form (10) holds. Finally, we can include a further component $\Lambda_{0}$ in (8), giving rise to 'dud runs' of the experiment.

Now take any run of Alice's measurement that has produced the result ' + '. The component $\rho_{\lambda}$ of $\rho$ that has led to this result is a state of the apparatus that will produce the result ' + ', if Alice couples it to her particle by the appropriate unitary interaction. Similarly, there exists another state of an apparatus that will produce the result '-', if Alice couples it to her particle by an appropriate unitary interaction. Of course, Alice cannot prepare these apparatus states in practice (she could prepare them in principle by reversing the time evolution). But we see that there exist states such that, by choosing to couple her particle to these states by an appropriate unitary interaction, Alice can produce at will any desired measurement outcome on her particle. But now, given (10), she can also guarantee that Bob will obtain the opposite result, if he measures the spin in the same direction on his own particle. Thus, Alice in principle can use this EPR setup to signal instantaneously to Bob.

But this is impossible: we know from the standard no-signalling theorem 
(Ghirardi, Rimini and Weber 1980) that Alice cannot signal instantaneously by performing local unitary operations on her side of an EPR setup.

It follows that using statistical mixtures of apparatus states to recover collapse behaviour - even if it were possible in the case of measurements on single particles - must fail to recover the collapse of the state of the distant particle in an EPR experiment.

\section{Einstein's 'insolubility' argument}

The above proof is extremely simple, and it would be quite remarkable if it were new. But I do not believe the proof is quite new. In fact, I see it essentially as a variant of the reasoning that Einstein developed in the run-up to the EPR paper (Einstein, Podolsky and Rosen 1935). This is perhaps not so evident from the text of the paper itself, but becomes much more so if one reads Einstein's own explanation of his reasoning to Schrödinger in their correspondence from the summer of $1935 .^{13}$

On 19 June 1935, Einstein writes to Schrödinger to thank him for his comments on the EPR paper, ${ }^{14}$ but explains that, since the actual paper had been written by Podolsky ('for language reasons'), what Einstein had actually wanted had 'not come out so well', and the main issue was 'so to speak buried in learnedness'.

Before turning to the quantum mechanical case, Einstein discusses a classical example consisting of two boxes that can be opened and inspected as to whether they contain a single ball (i. e. only one or the other of the boxes is

\footnotetext{
${ }^{13}$ Extracts of this correspondence are well-known from the discussions by Fine (1981) and by Howard $(1985,1990)$. The correspondence has now been published in full (in the original German) as part of the extensive selection of Schrödinger's correspondence edited by von Meyenn (2011). The following translations of passages from Einstein's letter of 19 June are by Elise Crull and myself. We are currently preparing a volume including commentary and translations of original materials relating to the 1935 debate on the EPR paper, focusing especially on some of its lesser known aspects (Bacciagaluppi and Crull, in preparation).

${ }^{14}$ Einstein to Schrödinger, 19 June 1935, Archive for the History of Quantum Physics, microfilm 92, section 2-107 (in German); von Meyenn (2011), vol. 2, pp. 537-539.
} 
found to be full on each run of the 'experiment'). He then asks whether the statement 'The probability that the ball is in the first box is $\frac{1}{2}$ ' is a complete description of the state of the system. If one denies it ('Bornian' view), one holds that the ball is in fact in one of the boxes, and the given state description is statistical. ${ }^{15}$ If one maintains that it is complete ('Schrödingerian' view), then the statistics of the measurement results are introduced only by the act of measurement. If one does not care about the reality of the ball ('Talmudist' view), this distinction between complete and incomplete descriptions is held to be meaningless. While in this everyday example the statistical view appears to be the only natural one, if one wishes to argue against the 'Talmudist', according to Einstein one needs a supplementary principle:

the second box, together with everything that pertains to its contents, is independent of what happens with respect to the first box (separate subsystems).

The argument so far, including Einstein's introduction of the 'separation principle', has been thoroughly and convincingly discussed in the existing literature - except for one small detail of Einstein's description of the 'Schrödingerian' view, which reads as follows, and to which we shall return later:

The statistics only come about through insufficiently known factors foreign to the described system, which are introduced by the observation.

Einstein then goes on to discuss the quantum mechanical case:

One describes in quantum theory a real state of a system by a (normalised) function $\psi$ of the coordinates (of configuration space). The time evolution is univocally given through the Schrödinger eq[uation]. One would now like to say the following: $\psi$ corresponds bi-univocally to the real state of the real system. The

\footnotetext{
${ }^{15}$ That is, it describes an ensemble of systems, half of which are in one state and half in the other.
} 
statistical character of the measurement outcomes is accounted for exclusively in terms of the measurement apparatus, or the process of measurement. If this works, I speak of a complete description of reality through the theory. If, however, such an interpretation is not feasible, I call the theoretical description 'incomplete'.

Note that what counts here for Einstein as a complete description in terms of quantum mechanics (which Einstein's argument aims to show is impossible) is a picture in which: (a) wave functions are in bijective correspondence with the real states of the system; (b) they evolve according to the unitary Schrödinger equation; (c) the statistical distribution of results is introduced only through the measurement process. ${ }^{16}$

Einstein then describes a generic EPR setup, with an entangled state

$$
\psi_{A B}=\sum_{m n} c_{m n} \psi_{m}\left(x_{1}\right) \chi_{n}\left(x_{2}\right)
$$

of two systems $A$ and $B$. Performing measurements of two alternative observables on subsystem $A$ yields different wave functions $\psi_{B}$ or $\psi_{B}$ for $B$. But, as Einstein observes:

The real state of $B$ now cannot depend on what measurement I perform on $A$ ('separation hypothesis' above).

Thus, the assumption of completeness contradicts the separation principle.

I suggest now that one could read the above condition (c) - that the statistics are introduced through the measurement process - in the same sense used by von Neumann: that (while all interactions are unitary) the exact details of the measurement process are unknown, and that it is for this reason that the results of the measurement are only statistically determined. This is after all the way measurement statistics are introduced in Einstein's

\footnotetext{
${ }^{16}$ One can perhaps say that a complete theory needs to describe not only the real states of a system, but also its dynamics (both when the system is isolated and when it is subject to measurement).
} 
parallel ball-in-a-box example, so the analogy is close at hand. If we read Einstein thus, ${ }^{17}$ then we can gloss the ensuing contradiction in terms of insolubility: under the assumption of bijective correspondence of wave functions to real states, unitary quantum mechanics cannot reproduce the full phenomenology of measurement by invoking an imperfectly known initial state of the apparatus (and is in this sense incomplete)!

Before concluding, it will be useful to examine two (related) aspects under which the 'insolubility' reading of Einstein's incompleteness argument and the argument given in the previous section are disanalogous. Einstein's argument considers two alternative measurements performed on subsystem $A$, while we considered the same kind of measurement, but with two different microscopic initial states for Alice's apparatus. Thus, on this reading, Einstein focuses on what Alice can do in practice, while our argument needs to invoke what she can do in principle. On the other hand, in our case the effect on Bob's side contradicts the no-signalling theorem, which follows from unitarity. In Einstein's case (precisely because the no-signalling theorem holds!) the effect on Bob's side cannot be an instance of signalling, and in order to derive a contradiction Einstein needs to invoke the full-blown separation principle. ${ }^{18}$

Einstein's separation principle in fact is so strong that it allows one to derive a contradiction whether or not one assumes a unitary interaction between Alice's system and her apparatus, or even whether or not one assumes that the evolution of the system upon measurement is deterministic. (This of

\footnotetext{
${ }^{17} \mathrm{I}$ am not claiming that this is the correct historical reading of Einstein's argument. Indeed, it is not an aspect that returns explicitly in later presentations of his incompleteness argument (although I see nothing in the later presentations that would exclude this reading). I am only suggesting that there is a possible analogy between certain aspects of Einstein's incompleteness argument and certain aspects of von Neumann's insolubility argument (and by extension certain aspects of our argument in section 3).

${ }^{18}$ According to Howard (1985), Einstein's separation principle is to be analysed in terms of separability (the existence of separate states) and locality (no superluminal influence), a distinction which Einstein starts making explicitly by 1946 or 1947. In terms of this distinction, another way of expressing the above disanalogy is to say that if one focuses on what Alice can do in principle, one can derive a contradiction with the weaker locality principle, while if one focuses on what Alice can do in practice, the contradiction will be only with the full separation principle. This may be a less anachronistic way of making the comparison, since as far as I am aware the no-signalling theorem was formulated explicitly only in 1980 by Ghirardi, Rimini and Weber.
} 
course helps to hide the analogy with von Neumann's insolubility argument, which refers explicitly to unitary interaction.)

Note finally that if one assumes a deterministic but nonlinear interaction between Alice's system and her measuring apparatus, then in order to derive signalling there is no need to consider what Alice could do in principle. As Gisin (1989) famously showed, if such a theory allows Alice to reproduce the collapse and Born rule of quantum mechanics also on Bob's side, then $B o b$ will be able in practice to use the nonlinearity in the Schrödinger equation to distinguish locally on his side between the different decompositions of the reduced state of his system corresponding to the different possible measurements performed by Alice. ${ }^{19}$

\section{Conclusion}

This paper was prompted by an engaging and provocative talk by Theo Nieuwenhuizen, ${ }^{20}$ presenting the model of spin measurements developed by Allahverdyan, Balian and Nieuwenhuizen (2011). At first, I thought this model was an attempt at doing precisely what is precluded by the insolubility results. A careful reading of the 160-page paper, however, makes it clear that the authors - in fact, like Einstein - reject an interpretation of the wave function as a complete description of an individual system. ${ }^{21}$ Moreover,

\footnotetext{
${ }^{19}$ The result is presented perhaps even more strikingly in Gisin (1990). It should be emphasised that (as Gisin realises perfectly well) the assumption from which signalling is derived, namely that a given theory with a nonlinear Schrödinger equation reproduce the standard predictions of quantum mechanics, is extremely strong and perhaps impossible to satisfy. See Doebner and Goldin (1996) and Bacciagaluppi (2012), respectively, for two explicit examples of nonlinear theories that do not exhibit signalling, or at least not for all entangled states.

${ }^{20}$ At the conference on 'Emergent Quantum Mechanics', University of Vienna, 11-13 November 2011.

${ }^{21}$ Under an alternative 'minimal' or 'statistical' interpretation of the theory - in the sense adopted by the above authors - the measurement problem could be said not to arise. Such an interpretation, however, throws open further weighty questions - of why and how in the context of macroscopic measurements we appear to have observational access to an individual description, and of whether and how this individual description extends also to a 'sub-quantum' level.
} 
the paper shows explicitly how an arguably realistic model of measurement ${ }^{22}$ does not exhibit the sensitivity to the state of the measuring apparatus that would be needed if one wished to explain the phenomenology of measurements through ignorance of the exact initial state of the apparatus. The mechanism that leads to the final mixture of pointer readings (irrespectively of the problem of how this mixture should be interpreted) is intuitively that the apparatus relaxes to different macroscopic equilibrium states for different eigenstates of the measured observable. Not only does this mechanism not depend on the exact initial microstate of the apparatus, but the authors show that the initial apparatus state can even be a pure state, which cannot be decomposed further (Allahverdyan, Balian and Nieuwenhuizen 2011, section 5.2). ${ }^{23}$ Thus, the kind of model that the insolubility results rule out does not even remotely resemble actual detailed models of the interaction between a system and a piece of apparatus.

\section{Acknowledgements}

I wish to thank in particular Arthur Fine for very perceptive comments on a previous draft of this paper. Many thanks also to Theo Nieuwenhuizen for inspiration, to Max Schlosshauer for correspondence, to two anonymous referees for shrewd observations, and to audiences at Aberdeen, Cagliari and Oxford (in particular to Harvey Brown, Elise Crull, Simon Saunders, Chris Timpson and David Wallace) for stimulating questions. This paper was written during my tenure of a Leverhulme Grant on 'The Einstein Paradox': The Debate on Nonlocality and Incompleteness in 1935 (Project Grant nr. F/00 152/AN), and it was revised for publication during my tenure of a Visiting Professorship in the Doctoral School of Philosophy and Epistemology, University of Cagliari (Contract nr. 268/21647).

\footnotetext{
${ }^{22}$ In particular one that incorporates the intuition that actual measuring apparatuses are typically macroscopic systems with typically thermal environments, and as such should be normally described by highly mixed states.

${ }^{23}$ Note that also in the classic model by Daneri, Loinger and Prosperi (1962) — which relies on the same intuitive mechanism - the initial state of the apparatus is explicitly taken to be pure.
} 


\section{References}

Allahverdyan, A. E., Balian, R., and Nieuwenhuizen, T. M. (2011), 'Understanding Quantum Measurement from the Solution of Dynamical Models', arXiv:1107.2138v1.

Bacciagaluppi, G. (2012), 'Non-equilibrium in Nelsonian Mechanics', Journal of Physics: Conference Series 361, 012017/1-12.

Bacciagaluppi, G., and Crull, E. M. (in preparation), 'The Einstein Paradox': The Debate on Nonlocality and Incompleteness in 1935 (Cambridge: Cambridge University Press, expected publication 2014 or 2015).

Bassi, A., and Ghirardi, G.C. (2000), 'A General Argument against the Universal Validity of the Superposition Principle', Physics Letters A 275, 373-381.

Bell, J. S. (1964), 'On the Einstein-Podolsky-Rosen Paradox', Physics 1, 195200. Reprinted in J. S. Bell, Speakable and Unspeakable in Quantum Mechanics (Cambridge: Cambridge University Press, 1987), pp. 14-21.

Brown, H. R. (1986), 'The Insolubility Proof of the Quantum Measurement Problem', Foundations of Physics 16, 857-870.

Busch, P., Grabowski, M., and Lahti, P. (1995), Operational Quantum Physics Lecture Notes in Physics, Vol. m31 (Berlin: Springer; second, corrected printing, 1997).

Busch, P., and Shimony, A. (1996), 'Insolubility of the Quantum Measurement Problem for Unsharp Observables', Studies in History and Philosophy of Modern Physics 27 B, 397-404.

Daneri, A., Loinger, A., and Prosperi, G. M. (1962), 'Quantum Theory of Measurement and Ergodicity Conditions', Nuclear Physics 33, 297-319.

Doebner, H.-D., and Goldin, G. A. (1996), 'Introducing Nonlinear Gauge Transformations in a Family of Nonlinear Schrödinger Equations', Physical Review A 54, 3764-3771.

Earman, J., and Shimony, A. (1968), 'A Note on Measurement', Il Nuovo Cimento B 54, 332-334.

Einstein, A., Podolsky, B., and Rosen, N. (1935), 'Can Quantum-Mechanical Description of Physical Reality be Considered Complete?', Physical Review 47, 777- 
780.

d'Espagnat, B. (1966), 'Two Remarks on the Theory of Measurement', Supplemento al Nuovo Cimento 4, 828-838.

d'Espagnat, B. (1971), Conceptual Foundations of Quantum Mechanics (Reading, Mass.: W. A. Benjamin).

Fehrs, M. H., and Shimony, A. (1974), 'Approximate Measurement in Quantum Mechanics. I', Physical Review D 9, 2317-2320.

Fine, A. (1970), 'Insolubility of the Quantum Measurement Problem', Physical Review D 2, 2783-2787.

Fine, A. (1981), 'Einstein's Critique of Quantum Theory: The Roots and Significance of EPR', in P. Barker and C. G. Shugart (eds.), After Einstein: Proceedings of the Einstein Centennial Celebration at Memphis State University, 14-16 March 1979 (Memphis: Memphis State University Press), pp. 147-158. Reprinted in A. Fine, The Shaky Game: Einstein, Realism and the Quantum Theory (Chicago: University of Chicago Press, 1986), pp. 26-39.

Gisin, N. (1989), 'Stochastic Quantum Dynamics and Relativity', Helvetica Physica Acta 62(4), 363-371.

Gisin, N. (1990), 'Weinberg's Non-linear Quantum Mechanics and Supraluminal Communications', Physics Letters A 143, 1-2.

Ghirardi, G.C., Rimini, A., and Weber, T. (1980), 'A General Argument against Superluminal Transmission through the Quantum Mechanical Measurement Process', Lettere al Nuovo Cimento 27(10), 293-298.

Howard, D. (1985), 'Einstein on Locality and Separability', Studies in History and Philosophy of Science 16, 171-201.

Howard, D. (1990), "Nicht sein kann was nicht sein darf", or the Prehistory of EPR, 1909-1935: Einstein's Early Worries about the Quantum Mechanics of Composite Systems', in A. I. Miller (ed.), Sixty-two Years of Uncertainty (New York: Plenum Press), pp. 61-111.

von Meyenn, K. (ed.) (2011), Eine Entdeckung von ganz außerordentlicher Tragweite: Schrödingers Briefwechsel zur Wellenmechanik und zum Katzenparadoxon, 
2 vols. (Berlin and Heidelberg: Springer).

von Neumann, J. (1932), Mathematische Grundlagen der Quantenmechanik (Berlin: Springer, 2nd ed. 1996). Transl. by R. T. Beyer as Mathematical Foundations of Quantum Mechanics (Princeton: Princeton University Press, 1955).

Price, H. (1996), Time's Arrow and Archimedes' Point: New Directions for the Physics of Time (New York: Oxford University Press).

Shimony, A. (1974), 'Approximate Measurement in Quantum Mechanics. II', Physical Review D 9, 2321-2323.

Stein, H. (1997), 'Maximal Extension of an Impossibility Theorem Concerning Quantum Measurement', in R. S. Cohen, M. Horne and J. Stachel (eds.), Potentiality, Entanglement and Passion-at-a-Distance: Quantum Mechanical Studies for Abner Shimony, Vol. 2, Boston Studies in the Philosophy of Science, Vol. 194 (Dordrecht: Kluwer), pp. 231-243.

Wigner, E. P. (1963), 'The Problem of Measurement', American Journal of Physics 31, 6-15. 